\title{
Quick Sequential Organ Failure Assessment Versus Systemic Inflammatory Response System in Early Detection of Sepsis in Emergency Department
}

\author{
Khaled MG Embaby ${ }^{1}$, Samir M Attia ${ }^{2}$, Amr S El-Saeed ${ }^{3}$, Hisham KH Ismael ${ }^{4}$ \\ Departments of ${ }^{1}$ Emergency Medicine, ${ }^{2}$ Vascular Surgery, ${ }^{3}$ General Surgery and ${ }^{4}$ Critical Care Medicine, \\ Faculty of Medicine, Mansoura University, Egypt \\ *Corresponding author: Hisham KH Ismael, Mobile: (+20)1095603422, E-Mail: Heshamismael69@ gmail.com
}

\begin{abstract}
:
Background: Sepsis is a life threating medical condition where infection leads to massive inflammatory response and eventually lead to organ dysfunction. It continues to pose a significant health threat despite remarkable developments in critical care medicine and extensive study of septic patients. Early recognition and treatment of sepsis in emergency department (ED) is important to reduce mortality, hospital length of stay and morbidity.

Objective: This study was aimed to validate the performance of qSOFA scoring system and SIRS criteria in early sepsis diagnosis in the Emergency Department.

Patient and method: This prospective observational clinical study was carried out in Emergency Department (ED) on 100 patients with suspected infection presented and admitted at o Mansoura University Emergency Hospital from February 2019 to February 2020. Patients were divided into two groups: infection group with qSOFA -ve criteria and sepsis group with qSOFA +ve group. We compare between qSOFA and SIRS scores in both groups.

Results: The study demonstrated that organ dysfunction >2, 2ry to infection (according to sepsis definition by sepsis3 task force) was more frequently reported among qSOFA + SIRS + group, compared to qSOFA - SIR - group. Moreover, both qSOFA and SIRS had comparable sensitivity (100\%) in prediction of mortality within 1 week, while qSOFA demonstrated higher specificity (53.3\%) in comparison with SIRS (20\%). Both scores had comparable sensitivity for prediction ICU admission and of mechanical ventilation (86.67 and 88.9) whereas qSOFA demonstrated higher specificity than SIRS for ICU admission (94.29 versus 71.43) as well as mechanical ventilation (82.9 versus 63.41).

Conclusion: It could be concluded that qSOFA is considered as specific not sensitive tool, while SIRS is more sensitive but not specific score for sepsis detection in emergency room.
\end{abstract}

Keywords: Sepsis, SIRS, qSOFA, Infection, ICU.

\section{INTRODUCTION}

Sepsis is a serious medical condition where infection leads to systemic inflammation and finally organ dysfunction. The estimated global incidence of hospital-treated sepsis and severe sepsis is 437 and 270, respectively, per 100,000 inhabitants. The incidence of sepsis and severe sepsis has increased in the last few decades, probably due to better recognition and increasing age. The incidence of sepsis is age-related with an increased incidence in both infants $(<1$ year) and the elderly ( $>65$ years). Mortality and long-term morbidity, especially among elderly patients with sepsis, is high ${ }^{(1)}$.

Since 1991, the Systemic Inflammatory Response Syndrome (SIRS) criteria have been used to classify sepsis. At an International Sepsis Definitions Conference in 2001 it was concluded that the SIRS criteria were too non-specific to diagnose systemic inflammation caused by an infection. However, due to the high sensitivity in predicting systemic inflammation, the SIRS criteria were maintained ${ }^{(2)}$.

As a result of growing criticism on the low specificity of the SIRS criteria, an update of the sepsis definition and criteria was needed. Early 2016; an international sepsis task force published a new international consensus for the definition of sepsis.
They defined sepsis as life-threatening organ dysfunction caused by a dysregulated host response to infection ${ }^{(3)}$.

Septic shock was defined as "a subset of sepsis in which particularly profound circulatory, cellular, and metabolic abnormalities are associated with a greater risk of mortality than with sepsis alone." Septic shock was diagnosed by identifying a vasopressor requirement to maintain a mean arterial pressure (MAP) $\geq 65 \mathrm{mmHg}$ and serum lactate concentration $>2 \mathrm{mmol} / \mathrm{L}$ despite adequate fluid resuscitation ${ }^{(4)}$.

The task force of sepsis-3 developed the more clinical, Quick Sequential Organ Failure Assessment (qSOFA) screening tool which is based on respiratory rate, systolic blood pressure and an altered mental state, which is an important part of the sepsis work-up in the Emergency Department ${ }^{(5)}$.

Most patients with sepsis are initially assessed in the ED. Because these patients present with a wide spectrum of disease courses and mortality risks and early recognition and treatment of them is important to reduce mortality, hospital length of stay and morbidity, an accurately predictive disease severity score is important, not only to predict mortality, but also to distribute resources appropriately or make decisions regarding resuscitation measures ${ }^{\left({ }^{(}\right)}$. 
This study was aimed to validate the performance of qSOFA scoring system and SIRS criteria in early sepsis diagnosis in the Emergency Department.

\section{PATIENT AND METHOD}

This prospective observational clinical study included a total of 100 patients with suspected infection, attending and admitted at Emergency Department (ED), Mansoura University Emergency Hospital. This study was conducted between February 2019 to February 2020.

\section{Ethical Consideration:}

This study was ethically approved by Ethical Committee, Faculty of Medicine, Mansoura University. Informed written consent was obtained from the patients sharing in the study or their relatives, confidentiality and personal privacy will be respected in all levels of the study, collected data will not be used for any other purpose.

\section{Inclusion Criteria:}

Patients with suspected infection based on clinical exam and necessary laboratory and radiologic evaluation of both genders and aged $>18$ years..

\section{Exclusion Criteria:}

Patients $<18$ years old, pregnant women, patients in cardio-respiratory arrest, and patients presented by trauma as main complaint in ED.

Patients were divided into two groups: infection group with qSOFA -ve criteria and sepsis group with qSOFA +ve criteria. comparison between qSOFA and SIRS scores in both groups was done.

All patients were subjected to complete history taking to identify patients with infection, full clinical examination, laboratory investigations: $\mathrm{CBC}, \mathrm{ABG}$, kidney function tests, liver function tests (AST, ALT, Bilirubin, Albumin), body fluids analysis (ascetic fluid, pleural fluid or CSF) if needed, serum lactate and acute phase reactant as CRP and radiological investigations: Chest X-ray, NCCT chest, abdominal Ultrasound, extremities Duplex Ultrasound and Echocardiography.

We apply SIRS and qSOFA scores: SIRS score included fever $>38.0^{\circ} \mathrm{C}$ or hypothermia $<36.0^{\circ} \mathrm{C}$, tachycardia $>90$ beats/minute, tachypnea $>20$ breaths/minute and leucocytosis $>12 * 10^{9} / 1$ or leucopoenia $<4 * 10^{9} / 1$. ${ }^{(7)}$, while qSOFA score included Respiratory rate $\geq 22 / \mathrm{min}$, Change in mental status and Systolic blood pressure $\leq 100 \mathrm{mmHg}{ }^{\left({ }^{8}\right)}$.

\section{Statistical analysis}

The collected data was revised, coded, processed and analyzed using SPSS program (Statistical Package for Social Science) for windows version 20 (SPSS Inc., Chicago, IL, USA). The data were presented as number and percentages for the qualitative data, mean, standard deviations and ranges for the quantitative data with parametric distribution and median with inter quartile range (IQR) for the quantitative data with non-parametric distribution. The appropriate tests of significance were conducted. Chi-square test was used in the comparison between the two groups with qualitative data and Fisher exact test was used instead of the Chi-square test when the expected count in any cell found less than 5 . Independent t-test was used in the comparison between the two groups with quantitative data and parametric distribution and Mann-Whitney test was used in the comparison between two groups with quantitative data and non-parametric distribution. $\mathrm{P}$ value $<0.05$ was considered significant.

\section{RESULTS}

Socio-demographics and clinical characteristics of study patients are shown in table 1 . The study included a total of 100 patients with a mean age of $58.2 \pm 17.9$ years. Of them, 50 were males and 50 were females. Comorbidities were reported in 90 patients. Past surgical history was present in 34 cases. Mean GCS was $14 \pm 2.4$.

The Site of infection, 30 patients had respiratory infections, 26 patients had abdominal infections, 40 patients had skin and soft tissue and 4 cases had infections in other sites. The median SBP and DBP of the study patients were $125 \mathrm{mmHg}$ and $70 \mathrm{mmHg}$, respectively. The mean pulse, RR and temperature were $94 \pm 15.8 \mathrm{BPM}, 22.2 \pm 7.7$ cycle/min and $37.4 \pm 0.9^{\circ} \mathrm{C}$, respectively. As regards the results, median WBCs were 14.2, median serum creatinine was 1.6, median Platelet count was 250.5, median was 1.5 and the mean paO2/F82 was $412 \pm$ 66.3. Also, positive culture was reported in 30 patients. 
Table (1): Sociodemographics and clinical characteristics of all patients:

\begin{tabular}{|c|c|}
\hline Parameter & $\mathbf{N}(\%) /$ Mean \pm SD \\
\hline Age (years) & $58.2 \pm 17.9$ \\
\hline Sex & \\
\hline Male & $50(50)$ \\
\hline Female & $50(50)$ \\
\hline Comorbidities & $90(90)$ \\
\hline Past surgical history & $34(34)$ \\
\hline Site of infection & \\
\hline Respiratory & $30(30)$ \\
\hline Abdominal & $26(26)$ \\
\hline Skin and soft tissue & $40(40)$ \\
\hline Others & 4 \\
\hline GCS & $14 \pm 2.4$ \\
\hline \multicolumn{2}{|l|}{ Vital signs } \\
\hline $\begin{array}{l}\text { SBP Median (min-max) } \\
\text { mmHg }\end{array}$ & $125(50-170)$ \\
\hline $\begin{array}{l}\text { DBP Median (min-max) } \\
\text { mmHg }\end{array}$ & $70(25-140)$ \\
\hline Pulse (beats per minute) & $94 \pm 15.8$ \\
\hline RR (breaths per minute) & $22.2 \pm 7.7$ \\
\hline Temparture $\left({ }^{\circ} \mathrm{C}\right)$ & $37.4 \pm 0.9$ \\
\hline \multicolumn{2}{|l|}{ Laboratory } \\
\hline $\begin{array}{l}\text { WBCs Median (min-max) } \\
\left(\mathrm{x} 10^{9} / \mathrm{l}\right)\end{array}$ & $14.2(4.5-29.6)$ \\
\hline $\begin{array}{l}\text { Serum creatinine Median } \\
(\min -m a x)(\mathrm{mg} / \mathrm{dl})\end{array}$ & $1.6(0.3-16.5)$ \\
\hline $\begin{array}{l}\text { Platelet Median (min-max) } \\
\left(\mathrm{x} 10^{9} / \mathrm{l}\right)\end{array}$ & $250.5(28-665)$ \\
\hline $\begin{array}{l}\text { Bilirubin Median (min- } \\
\max )(\mathrm{mg} / \mathrm{dl})\end{array}$ & $1.2(0.4-4.1)$ \\
\hline paO2/F82 & $412 \pm 66.3$ \\
\hline Culture (positive) & $30(30)$ \\
\hline
\end{tabular}

qSOFA ,SIRS and outcomes of studied patients are shown in table 2. qSOFA zero was the most frequent and it was reported in nearly half of the patients (48 patients), qSOFA 1 was reported in 22 patients, qSOFA 2 was reported in 16 patients while qSOFA 3 was the least frequently reported (in 14 patients).

SIRS zero was reported in 18 patients, SIRS 1 was the most frequent and it was reported in 36 patients, SIRS 2 was reported in 22 patients, SIRS 3 was reported in 18 patients and SIRS 4 was the least frequently reported (in 6 patients). Organ dysfunction $\leq 2$ was reported most of patients (70 patients) while Organ dysfunction $>2$ was reported in 30 patients. Thirty patients were admitted to ICU with a mean ICU length of stay of 4 days. The mean ward length of stay was also 4 days. Ten cases died within one week. Twenty-six patients were put on vasopressors while 18 patients required ventilator.

Table (2): qSOFA, SIRS and outcomes of studied patients:

\begin{tabular}{|l|c|}
\hline Parameter & $\begin{array}{c}\text { N (\%) / Median } \\
\text { (min-max) }\end{array}$ \\
\hline qSOFA & $48(48)$ \\
$\mathbf{0}$ & $22(22)$ \\
$\mathbf{1}$ & $16(16)$ \\
$\mathbf{3}$ & $14(14)$ \\
\hline SIRS & $18(18)$ \\
$\mathbf{0}$ & $36(36)$ \\
$\mathbf{1}$ & $22(22)$ \\
$\mathbf{2}$ & $18(18)$ \\
$\mathbf{3}$ & $6(6)$ \\
$\mathbf{4}$ & \\
\hline Organ dysfunction & $70(70)$ \\
$\mathbf{3} \mathbf{2}$ & $30(30)$ \\
\hline $\mathbf{2}$ & \\
\hline & $30(30)$ \\
\hline ICU admission & $4(1-10)$ \\
\hline ICU length of stay & $4(1-10)$ \\
\hline Ward length of stay & \\
\hline Mortality within 1 week & $10(10)$ \\
\hline Vasopressor & $26(26)$ \\
\hline Ventilator & $18(18)$ \\
\hline
\end{tabular}

As shown in table 3 , Organ dysfunction $\geq 2$ was more frequently reported among qSOFA positive than qSOFA negative (22 cases versus 8 cases) with a statistically significant difference. Similarly, ICU admission was more frequently reported among qSOFA positive than qSOFA negative ( 26 cases versus 4 cases) with a statistically significant difference. No statistically significant difference existed between qSOFA positive and qSOFA negative cases as regards length of ICU stay, length of ward stay or mortality within 1 week. Use of Vasopressor and Ventilator was more evident among qSOFA positive cases compared with qSOFA negative cases (24 versus 2 and 16 versus 2 for Vasopressor and Ventilator, respectively) with a statistically significant difference. 
Table (3): qSOFA, SIRS and outcomes of qSOFA positive and qSOFA negative:

\begin{tabular}{|l|c|c|c|}
\hline \multirow{2}{*}{ Parameter } & qSOFA positive (n=30) & qSOFA negative (n=70) & \multirow{2}{*}{ P value } \\
\cline { 2 - 4 } & \multicolumn{2}{|c|}{$\mathbf{N}(\mathbf{\%}) /$ Median (Min-Max) } & \\
\hline Organ dysfunction $\geq \mathbf{2}$ & $22(73.3)$ & $8(11.4)$ & $\leq 0.001$ \\
\hline ICU admission & $26(86.7)$ & $4(5.7)$ & $\leq 0.001$ \\
\hline ICU stay (days) & $3(1-10)$ & $4.5(4-5)$ & 0.2 \\
\hline Ward stay (days) & $4(1-10)$ & $4(1-10)$ & 0.6 \\
\hline Mortality within 1 week & $6(20)$ & $4(5.7)$ & 0.03 \\
\hline Vasopressor & $24(80)$ & $2(2.9)$ & $\leq 0.001$ \\
\hline Ventilator & $16(53.3)$ & $2(2.9)$ & $\leq 0.001$ \\
\hline
\end{tabular}

As shown in table 4, Organ dysfunction $>2$ was more frequently reported among SIRS positive than SIRS negative (22 cases versus 8 cases) with a statistically significant difference. Similarly, ICU admission was more frequently reported among SIRS positive than SIRS negative (26 cases versus 4 cases) with a statistically significant difference. No statistically significant difference existed between SIRS positive and SIRS negative cases as regards length of ICU stay, length of ward stay or mortality within 1 week. Use of Vasopressor and Ventilator was more evident among SIRS positive cases compared with SIRS negative cases (24 versus 2 and 16 versus 2 for Vasopressor and Ventilator, respectively) with a statistically significant difference.

Table (4): qSOFA, SIRS and outcomes of SIRS positive and SIRS negative:

\begin{tabular}{|c|c|c|c|}
\hline \multirow[t]{2}{*}{ Parameter } & SIRS positive $(n=46)$ & SIRS negative $(n=54)$ & \multirow[t]{2}{*}{$\mathrm{P}$ value } \\
\hline & \multicolumn{2}{|c|}{ N (\%) / Median (Min-Max) } & \\
\hline Organ dysfunction >2 & $22(47.8)$ & $8(14.8)$ & $\leq 0.001$ \\
\hline ICU admission & $26(56.6)$ & $4(7.4)$ & $\leq 0.001$ \\
\hline ICU stay (days) & $3(1-10)$ & $5(5-5)$ & 0.05 \\
\hline Ward stay (days) & $4(1-10)$ & $4(1-10)$ & 0.9 \\
\hline Mortality within 1 week & $6(13)$ & $4(7.4)$ & 0.3 \\
\hline Vasopressor & $24(52.2)$ & $2(3.7)$ & $\leq 0.001$ \\
\hline Ventilator & $16(34.8)$ & $2(3.7)$ & $\leq 0.001$ \\
\hline
\end{tabular}

As shown in table 5, Organ dysfunction $>2$ was more frequently reported among qSOFA +and SIRS + $(20$ cases) compared with qSOFA + and SIRS - (2 cases), qSOFA -and SIRS + (2 cases) and qSOFA -and SIRS - (6 cases) with a statistically significant difference. ICU admission was more frequently reported among qSOFA +and SIRS + (24 cases), compared with qSOFA + and SIRS - ( 2 cases), qSOFA - and SIRS + ( 2 cases) and qSOFA -and SIRS - ( 2 cases) with a statistically significant difference. No statistically significant difference existed between qSOFA + and SIRS+, compared with qSOFA + and SIRS -, qSOFA- and SIRS + and qSOFA-and SIRS - as regards length of ICU stay, length of ward stay or mortality within 1 week. Use of Vasopressor was more evident among qSOFA + and SIRS + cases (24 cases) compared with only 2 cases of qSOFA -and SIRS -cases. Vasopressor was not used in qSOFA + and SIRS - cases or qSOFA -and SIRS + cases. A statistically significant difference existed between groups. Ventilator was more frequently used among qSOFA + and SIRS + (14 cases) compared with qSOFA + and SIRS - cases ( 2 cases) and qSOFA -and SIRS + cases ( 2 cases) while it was never used among qSOFA-and SIRS - cases. A statistically significant difference existed between groups.

Table (5): Comparison of outcomes according to both qSOFA and SIRS:

\begin{tabular}{|l|c|c|c|c|c|}
\hline \multirow{2}{*}{ Parameter } & $\begin{array}{c}\text { qSOFA+ and } \\
\text { SIRS + } \\
\text { n=26 }\end{array}$ & $\begin{array}{c}\text { qSOFA+ and } \\
\text { SIRS - } \\
\mathbf{n = 4}\end{array}$ & $\begin{array}{c}\text { qSOFA-and } \\
\text { SIRS + } \\
\text { n=20 }\end{array}$ & $\begin{array}{c}\text { qSOFA-and } \\
\text { SIRS- } \\
\text { n=50 }\end{array}$ & P value \\
\cline { 2 - 5 } & \multicolumn{5}{|c|}{ N (\%) / Median (Min-Max) } \\
\hline Organ dysfunction >2 & $20(76.9)$ & $2(50)$ & $2(10)$ & $6(12)$ & $\leq 0.001$ \\
\hline ICU admission & $24(92.3)$ & $2(50)$ & $2(10)$ & $2(4)$ & $\leq 0.001$ \\
\hline ICU stay (days) & $3(1-10)$ & $5(5-5)$ & $4(4-4)$ & $5(5-5)$ & 0.2 \\
\hline Ward stay (days) & $4(1-10)$ & $2(1-3)$ & $3.5(1-6)$ & $4(1-10)$ & 0.2 \\
\hline Mortality within 1 week & $4(15.4)$ & $2(50)$ & $2(10)$ & $2(4)$ & 0.02 \\
\hline Vasopressor & $24(92.3)$ & - & - & $2(4)$ & $\leq 0.001$ \\
\hline Ventilator & $14(53.8)$ & $2(50)$ & $2(10)$ & - & $\leq 0.001$ \\
\hline
\end{tabular}

As shown in table 6 , a Cut off value of qSOFA $>1$ was associated with $86.67 \%$ sensitivity and $71.43 \%$ specificity for prediction of ICU admission. The AUC was 0.964. A Cut off value of SIRS $>1$ was associated with $86.67 \%$ sensitivity and $94.29 \%$ specificity for prediction of ICU admission. The AUC was 0.834 . 
Table (6): AUC and diagnostic value of qSOFA for prediction of ICU admission:

\begin{tabular}{|l|c|c|}
\hline Parameters & qSOFA & SIRS \\
\hline AUC & $\mathbf{0 . 9 6 4}$ & $\mathbf{0 . 8 3 4}$ \\
\hline Cut off value & $>1$ & $>1$ \\
\hline Sensitivity $(95 \%$ CI) & $\mathbf{8 6 . 6 7}(69.3-96.2)$ & $86.67(69.3-96.2)$ \\
\hline Specificity $(95 \%$ CI) & $94.29(86.0-98.4)$ & $71.43(59.4-81.6)$ \\
\hline PPV $(95 \%$ CI) & $\mathbf{8 6 . 7}(69.3-96.2)$ & $92.6(82.1-97.9)$ \\
\hline NPV $(95 \%$ CI) & $94.3(86.0-98.4)$ & $56.5(41.1-71.1)$ \\
\hline
\end{tabular}

As shown in table 7, a Cut off value of qSOFA >0 was associated with $100 \%$ sensitivity and $53.3 \%$ specificity for prediction of mortality within 1 week. The AUC was 0.76.A Cut off value of SIRS $>0$ was associated with $100 \%$ sensitivity and $20 \%$ specificity for prediction of mortality within 1 week. The AUC was 0.627 .

Table (7): AUC and diagnostic value of qSOFA for prediction of mortality within 1 week:

\begin{tabular}{|l|c|c|}
\hline Parameters & qSOFA & SIRS \\
\hline AUC & 0.76 & 0.627 \\
\hline Cut off value & $>0$ & $>0$ \\
\hline Sensitivity (95\% CI) & $100(69.2-100)$ & $100(69.2-100)$ \\
\hline Specificity (95\% CI) & $\mathbf{5 3 . 3 ( 4 2 . 5 - 6 3 . 9 )}$ & $\mathbf{2 0}(12.3-29.8)$ \\
\hline PPV $(95 \%$ CI) & $19.2(9.6-32.5)$ & $12.2(6-21.3)$ \\
\hline NPV $(95 \%$ CI) & $100(92.6-100)$ & $100(81.5-100)$ \\
\hline
\end{tabular}

As shown in table 8, a cut off value of qSOFA $>1$ was associated with $88.9 \%$ sensitivity and $82.9 \%$ specificity for prediction of need to mechanical ventilator. The AUC was 0.917 . A Cut off value of SIRS $>1$ was associated with $88.9 \%$ sensitivity and $63.41 \%$ specificity for prediction of need to mechanical ventilator. The AUC was 0.839 .

Table (8): AUC and diagnostic value of qSOFA for prediction of need to mechanical ventilator:

\begin{tabular}{|l|c|c|}
\hline Parameters & qSOFA & SIRS \\
\hline AUC & $\mathbf{0 . 9 1 7}$ & $\mathbf{0 . 8 3 9}$ \\
\hline Cut off value & $>1$ & $>1$ \\
\hline Sensitivity (95\% CI) & $\mathbf{8 8 . 9}(65.3-98.6)$ & $\mathbf{8 8 . 9}(65.3-98.6)$ \\
\hline Specificity (95\% CI) & $\mathbf{8 2 . 9 ( 7 3 - 9 0 . 3 )}$ & $63.41(52-73.8)$ \\
\hline PPV (95\% CI) & $\mathbf{5 3 . 3 ( 3 4 . 3 - 7 1 . 7 )}$ & $\mathbf{3 4 . 8 ( 2 1 . 4 - 5 0 . 2 )}$ \\
\hline NPV (95\% CI) & $\mathbf{9 7 . 1 ( 9 0 . 1 - 9 9 . 7 )}$ & $\mathbf{9 6 . 3 ( 8 7 . 3 - 9 9 . 5 )}$ \\
\hline
\end{tabular}

\section{DISCUSSION}

In the current study; qSOFA, SIRS and general outcomes of studied patients, qSOFA zero was the most frequent and it was reported in the majority of cases $(48 \%)$ followed by, qSOFA $1(22 \%)$ and qSOFA 2 (16\%) and finally qSOFA $3(14 \%)$. As regards SIRS, most of them had score one then two followed by zero and 4 . Organ dysfunction $\leq 2$ was reported in most of patients (70 patients) while Organ dysfunction $>2$ was reported in 30 of which. Thirty patients were admitted to ICU with a mean ICU length of stay of 4 days. The mean ward length of stay was also 4 days. Ten cases died within one week. Twentysix patients were put on vasopressors while 18 patients required ventilator.

Similarly, Loritz et al. (9) conducted a prospective single-center study including patients presenting to the ED of any non-traumatic cause.
Primary outcome was development of sepsis within 48 hours; secondary outcomes were 30-day mortality and ICU stay for $>3$ days. Data were collected within one hour after arrival to indicate an impression of initial medical contact. Among 1,668 patients, 105 sepsis cases were identified. $8.4 \%$ presented with qSOFA $\geq$ $2,27.2 \%$ with SIRS $\geq 2$ within one hour. They demonstrated that, the median hospital length of stay was 4 days (IQR 1-8) and 30-day mortality was 3.1\% overall.

Likewise, Askim et al. ${ }^{(10)}$ conducted an observational cohort study performed at one ED in an urban university teaching hospital in Norway, with approximately 20,000 visits per year. All patients $>16$ years presenting with symptoms or clinical signs suggesting an infection $(n=1535)$ were prospectively included in the study from January 1 to December 31, 2012 reported that $17(2.6 \%)$ patients with sepsis died 
within 7 days and $42(6.3 \%)$ within 30 days. Eight (7.4\%) patients with severe sepsis died within seven days and 19 (17.6\%) died within 30 days.

Comparably, Churpek et al. ${ }^{(11)}$ reported that only $9 \%$ of the 30,667 patients admitted to an ED or a ward with infection suspicion had a qSOFA $\geq 2$ at time of presentation and that the qSOFA only had $29.9 \%$ sensitivity for detecting organ dysfunction according to the sepsis-3 task force definition of sepsis.

Socio-demographic and clinical characters in relation to qSOFA and SIRS, the current study demonstrated that there were statistically significant differences between cases with positive and negative SOFA and SIRS as regards sex and bilirubin $(\mathrm{P}<0.05)$ and highly statistically significant differences as regards site of infection, GCS, SBP, DBP, Pulse, RR $\mathrm{paO} 2 / \mathrm{Fio} 2(\mathrm{P} \leq 0.001)$, while there were no statistically significant differences among both groups as regards age, comorbidities, past surgical history, temperature, WBCs, serum creatinine, platelet count and positivity of culture $(\mathrm{P}>0.05)$.

Similarly, Loritz et al. ${ }^{(9)}$ demonstrated that there were highly statistically significant differences among cases with positive and negative SOFA as regards GCS, SBP, DBP, Pulse, RR and confirmed infection $(\mathrm{P} \leq 0.001)$. However, they were in disagreement with present study, as they demonstrated also that there were highly statistically significant differences as regards temperature and WBCs $(\mathrm{P} \leq 0.001)$.

The present study demonstrated that Organ dysfunction $>2$ was more frequently reported among qSOFA+\& SIRS+ compared with qSOFA+\& SIRS-, qSOFA-\& SIRS+ and qSOFA-\& SIRS - with a statistically significant difference. ICU admission was more frequently reported among qSOFA+\& SIRS, compared with qSOFA+\& SIRS-, qSOFA-\& SIRS+ and qSOFA-\& SIRS - with a statistically significant difference. No statistically significant difference existed between qSOFA+\& SIRS + compared to with qSOFA+\& SIRS-, qSOFA-\& SIRS+ and qSOFA-\& SIRS - as regards length of ICU stay, length of ward stay or mortality within 1 week.

In the same line Loritz et al. ${ }^{\left({ }^{(9)}\right.}$ demonstrated that there were highly statistically significant differences among cases with positive than negative SOFA as regards hospital stay, ICU admission, 30 day mortality ( $\mathrm{P} \leq 0.001)$.

Likewise, Boillat et al. ${ }^{(12)}$ revealed that the mortality of patients with a SOFA $\geq 2$ was $15 \%$, which also is in line with the anticipated overall mortality of around $10 \%$ in this subset of patients according to the new Sepsis-3 definition.

Similarly, Anand et al. (13) demonstrated that of 1,004,347 hospitalized patients, 271,500 (27.0\%) were qSOFA-positive on admission. In comparison with qSOFA-negative patients, qSOFA-positive patients needed ICU admission more often (28.5\% vs $6.5 \%)$, and had higher rate of mortality (6.7\% vs $0.8 \%)$

The current study demonstrated that both qSOFA and SIRS had comparable sensitivity (100\%), while qSOFA demonstrated higher specificity (53.3\%) in comparison with SIRS (20\%) in prediction of mortality within 1 week (cut off less than zero). Moreover, both scores had comparable sensitivity for prediction ICU admission and of mechanical ventilator (86.67 and 88.9). qSOFA demonstrated higher specificity than SIRS for ICU admission (94.29 versus $71.43)$ as well as mechanical ventilator (82.9 versus 63.41).

Boillat-Blanco et al. ${ }^{(12)}$ revealed that, the prognostic accuracy of qSOFA (AUROC $0.80,95 \%$ CI $0.73-0.87$ ) for 28-day mortality was similar to SOFA (AUROC 0.79, 0.71-0.87; $\mathrm{p}=0.1$ ) and better than SIRS with highly statistically significant difference (AUROC 0.61, 0.52-0.71; p<0.001). However, SIRS criteria had a higher sensitivity (91\%) compared to qSOFA score (59\%) in detecting sepsis.

Similarly, Loritz et al. (9) revealed that, sensitivity of qSOFA in predicting sepsis was lower compared to the SIRS criteria. In addition, qSOFA showed better prognostic accuracy for 30-day mortality compared to SIRS $(\mathrm{p}<0.05)$, but not for prolonged ICU stay $(\mathrm{p}=0.56)$ with a significantly higher AUC. qSOFA showed poorer results compared to the SIRS criteria as regard prolonged ICU stay, which is in disagreement with the current study

Comparably, Monclús et al. ${ }^{(14)}$ even report qSOFA $\geq 2$ to be the only independent variable associated with mortality. However, it should be noted that the reason for the high AUC lies foremost in its excellent specificity $(94.3 \%)^{(15)}$.

In parallel, Peneva et al. ${ }^{(16)}$ reported that both SIRS and qSOFA criteria used for early detection of sepsis are useful clinical tools for mortality predictability in septic patients. Cut-off values for SIRS higher than 2.5 points showed $91 \%$ sensitivity and $60 \%$ specificity - (AUC $0.80,95 \%$ CI - 0.712 0.907), whereas qSOFA scores greater than 1.5 points indicated sensitivity of $82.2 \%$ and specificity of $70.3 \%$ (AUC 0.85, 95\% CI 00.770 -0.934).

Ortega et al. ${ }^{(17)}$, reported that qSOFA offered high specificity for the prediction of sepsis and adverse outcomes however poor sensitivity in comparison with SIRS. There were 2523 cases included in the analysis and $39(1.6 \%)$ had the primary outcome of sepsis. The area under the curve for sepsis was 0.79 (95\% confidence interval [CI] 0.71-0.86) for qSOFA, 0.81 (95\% CI 0.73-0.87) for SIRS.

\section{CONCLUSION}

It could be concluded that qSOFA is considered as specific not sensitive tool, while SIRS is more sensitive but not specific score for sepsis detection in emergency room. In addition, they cannot be used as 
reliable indicators for sepsis, but they have the ability to give a very good impression for the prognosis and outcomes of cases in terms of prediction of ICU admission, mortality within 1 week and mechanical ventilator especially if used together.

\section{REFERENCES}

1. Fleischmann C, Scherag A, Adhikari N et al. (2016): Assessment of global incidence and mortality of hospitaltreated sepsis. Current estimates and limitations. American Journal of Respiratory and Critical Care Medicine, 193(3): 259-272.

2. Levy M, Fink M, Marshall J et al. (2003): 2001SCCM/ESICM/ACCP/ATS/ SIS International Sepsis Definitions Conference. Intensive Care Med., 29:530-8.

3. Singer M, Deutschman C, Seymour C et al. (2016): The Third International Consensus Definitions for Sepsis and Septic Shock (Sepsis-3): JAMA., 315:801-10.

4. Vincent J, Opal S, Marshall J et al. (2013): Sepsis definitions: time for change. Lancet (London, England), 381(9868): 774-8.

5. Vincent J, Martin G, Levy M (2016): qSOFA does not replace SIRS in the definition of sepsis. Crit Care, 20:2105.

6. Hutchison R, Govathoti D, Fehlis K et al. (2011): Improving severe sepsisoutcomes: cost and time to first antibiotic dose. Dimensions of Critical Care Nursing, 30(5): 277-282.

7. Alsulaiman D, Kubiak D (2017): Criteria for Sepsis: Systemic Inflammatory Response Syndrome (SIRS) and Quick Sepsis-Related Organ Dysfunction Assessment (QSOFA). Current Emergency and Hospital Medicine Reports, 5, 28-32.

8. Singer M, Deutschman C, Seymour C et al. (2016): The Third International Consensus Definitions for Sepsis and Septic Shock. JAMA., 315(8):801-10.

9. Loritz M, Busch H, Helbing T et al. (2020): Prospective evaluation of the quickSOFA score as a screening for sepsis in the emergency department. Internal and Emergency Medicine, 15: 685-693.

10. Askim A, Moser F, Gustad L et al. (2017): Poor performance of quick-SOFA (qSOFA) score in predicting severe sepsis and mortality-a prospective study of patients admitted with infection to the emergency department. Scandinavian Journal of Trauma, Resuscitation and Emergency Medicine, 25(1): 56-9.

11. Churpek M, Snyder A, Han X et al. (2016): qSOFA, SIRS, and early warning scores for detecting clinical deterioration in infected patients outside the ICU. Am J Respir Crit Care Med., 195(7): 906-911.

12. Boillat-Blanco N, Mbarack Z, Samaka J et al. (2018): Prognostic value of quickSOFA as a predictor of 28-day mortality among febrile adult patients presenting to emergency departments in Dar es Salaam, Tanzania. PloS One, 13(6): 197982-6.

13. Anand $V$, Zhang Z, Kadri S et al. (2019): Epidemiology of quick sequential organ failure assessment criteria in undifferentiated patients and association with suspected infection and sepsis. Chest, 156(2): 289-297.

14. Monclús $\mathrm{C}$, Ugarte $\mathrm{R}$, Gabara $\mathrm{X}$ et al. (2018): Comparison of the Quick Sepsis-related Organ Dysfunction score and severity levels assigned with the Andorran Triage Model in an urban tertiary care hospital emergency department. Emergencias: revista de la Sociedad Espanola de Medicina de Emergencias, 30(6): 400-6.

15. Schmoch T, Bernhard M, Uhle F et al. (2017): New sepsis-3 definition: do we have to treat sepsis before we can diagnose it from now on? Anaesthesist, 66(8):614-621

16. Peneva $P$, Nikolova $S$, Bocheva $Y$ (2019): Which rating system is better-qSOFA or SIRS? Scripta Scientifica Medica., 51 (4): 19-25.

17. Ortega $\mathbf{R}$, Rosin $\mathrm{C}$, Bingisser $\mathbf{R}$ et al. (2019): Clinical scores and formal triage for screening of sepsis and adverse outcomes on arrival in an emergency department all-comer cohort. The Journal of Emergency Medicine, 57(4): 453460. 